\title{
Research Paper \\ Effectiveness of Reward-Based Task on Affective Levels of Depressed Individuals
}

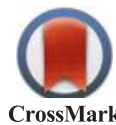

\author{
*Alireza Karimpour Vazifehkhorani ${ }^{1}$, Abbas Bakhshipour Roodsari ${ }^{2}$, Hossein Kamali Ghasemabadi ${ }^{1}$, Niloofar Etemadi \\ Chardah ${ }^{1}$
}

1. MSc., Department of Psychology, Faculty of Education and Psychology, Tabriz university, Tabriz, Iran

2. PhD in Psychology, Professor, Department of Psychology, Faculty of Education and Psychology, Tabriz university, Tabriz, Iran.

Received: 08 Jun. 2017

Accepted: 11 Oct. 2017

Key words:

Depression

Reinforcement,

Reward-based task,

Affective levels
Citation: Karimpour Vazifehkhorani A, Bakhshipour Roodsari A, Kamali Ghasemabadi H, Etemadi Chardah N. [Effectiveness of Reward-Based Task on Affective Levels of Depressed Individuals (Persian)]. Iranian Journal of Psychiatry and Clinical Psychology. 2018; 24(1):6-15. https://doi.org/10.29252/NIRP.IJPCP.24.1.6.

doil": https://doi.org/10.29252/NIRP.IJPCP.24.1.6

\begin{abstract}
A B S T R A C T
Objectives The present study examined the effects of reward-driven task on improving the affective levels in individuals with depressive symptoms.

Methods The present study is an experiment study with pretest- posttest and follow-up with control group. The community of this research was the students in Tabriz University in 2016-2017 semester. The sample size was 40 students which had visited the university counseling center 20 of them were assigned randomly to control group and 20 other experimental group. The intervention was given to the experimental group during 8 sessions ( 45 minutes per session) and 25 days by the Balloon Analogue Risk (reward-based task test), and the equivalent score obtained in each session was given to material rewards. Beck Depression Scale (BDI-II) and Mood Adjective Check List (MACL) were used for data collection. For data analysis, covariance analysis and SPSS version 23 software were used.

Results The posttest mean scores of the participants based on reward-based task in the intervention group were 18.1, 12.6, and 14.1 for hedonic tone, tense arousal, and energetic arousal, respectively, and 13.9, 19.3, and 13.2, respectively for the control group; the difference between the two groups was statistically significant $(P=0.01)$. The effect size of the present intervention was 0.70 . In addition, the observed difference between the mean scores of the affective levels in the participants based on the reward-based task in the intervention group (mean scores 15.7, 14.4, and 11.7 for hedonic tone, tense arousal, and energetic arousal, respectively) and the control group (mean scores 14.1, 18.9, and 13.6 for hedonic tone, tense arousal, and energetic arousal, respectively) differed significantly in the follow-up stage $(\mathrm{P}<0.01)$. The provision of the reward-based task had been effective in improving the affective levels of the participants of the intervention group in posttest and follow-up stages, and statistical power equaling 1.0 indicated a reliable statistical accuracy.

Conclusion Rewards and consequences of fun-producing factor strengthened the desire to earn rewards and improve the activity levels that would affect the depressed patients, and the negative effect was reduced with the reward situation.
\end{abstract}




\section{Extended Abstract}

\section{Introduction}

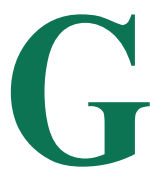

aining reward and pleasure is one of the major factors among individuals with depression, the lack of which leads to issues with respect to the affective levels in depressed individuals, and consequently, the defect in earning rewards, would lead to decreased activity and social reinforcement and increased negative thoughts, affective level, and motivation. This phenomenon would form a vicious cycle that would lead to the continuation of the depression disorder. Also, since rewards are essential for betterment, they are desirable and sought after. Therefore, using rewards creates enjoyable effects, and this factor leads to consolidation of a tendency towards using rewards. Yet the depressed individuals, because of deprivation of sensory mechanisms of pleasure in brain, cannot enjoy normal rewards and daily activities. On the other hand, based on the behavioral theory, depression is a chain of loss, decrease, lack of reward, and inability to earn rewards in interpersonal relationships. Therefore, the present study was aimed at investigating the effect of reward-based tasks on affective levels in depressed individuals.

\section{Method}

The present study used an interventional method and the cohort comprised of students of Tabriz University in the academic year of 2016-2017. The sample size included 40 students, who had referred to the University counseling center, were divided into two groups of control and intervention ( $\mathrm{n}=20$ per group) through random assignment method. The intervention was given to the experimental group during 8 sessions (45 minutes per session) and 25 days by the Balloon Analogue Risk (reward-based task test), and the equivalent score obtained in each session was given to material rewards. For data assimilation, the Beck Depression Inventory (BDI) and Mood Adjective Check List (MACL) were used.

\section{Results}

The posttest mean scores of participants based on the reward-based task in the intervention group were 18.1, 12.6, and 14.1 for hedonic tone, tense arousal, and energetic arousal, respectively, and 13.9, 19.3, and 13.2, respectively for the control group with significant differences $(\mathrm{P}=0.01)$. The effect size of the present intervention was 0.70 . In addition, the observed difference between the mean scores of the affective levels in the participants based on the reward-based task in the intervention group (by mean scores of 15.7, 14.4, and 11.7 for hedonic tone, tense arousal, and energetic arousal, respectively) and the control group (by mean scores of 14.1, 18.9, and 13.6 for hedonic tone, tense arousal, and energetic arousal, respectively) was significant in the follow-up stage $(\mathrm{P}<0.01)$. The provision of the reward-based task had been effective in improving the affective levels of participants in the intervention group in posttest and follow-up stages, and a statistical power equaling 1.0 indicated a reliable statistical accuracy.

\section{Discussion}

Assigning the reward-based task to individuals with depression symptoms led to an increase in hedonic tone. According to Frester's model, depression consists of a chain of loss, reduction or lack of reward, and inability to gaining rewards. Thus, depression is the cause of failure to achieve reinforcers, and until this vicious circle continues, depression would stay stable; therefore, depressed individuals can be aided via improvement of their affective levels by offering rewards. In addition, offering the reward-based task to individuals with depression disorder symptoms leads to an increase in their energetic arousal. Furthermore, negative affection is reduced through reward-gaining condition and is also increased through punishment condition. Individuals feel pleasure and convenience when faced with reward, while uncomfortable and nervous in the event of a punishment condition. In addition, offering a reward-based task to individuals with depressive symptoms in this study led to a reduction in tension arousal, thereby indicating that reward and punishment are significant sources for every behavior, and typically, individuals act such as to gain reward and avoid punishment.

Overall, in the present study, we expected that the affective level of the participants would become positive in reward-gaining condition and would also perform the assigned task better in the reward-gaining condition than in the punishment condition. This expectation was largely confirmed, and the negative affection was reduced in the reward condition. Gaining reward creates pleasurable consequences, which leads to the consolidation of the desire and act in order to achieve reward, further leading to the improvement in affective levels among depressed individuals and reduction of negative affection in punishment condition. Therefore, psychotherapists are recommended to utilize rewards and reinforcers in the treatment of individuals with depression in order to improve their mood and affective 
levels as this enhances the effectiveness of therapeutic techniques on these individuals.

\section{Ethical Considerations}

\section{Compliance With Ethical Guideline}

After expressing the research goals for the partners, their satisfaction was gained to participate in the research, and they were assured of the confidentiality of the information and the eligibility to participate in the research. After the end of the study, in order to observe ethical considerations, the control group also intervened.

\section{Funding}

This research did not receive any specific grant from funding agencies in the public, commercial, or not-forprofit sectors.

\section{Conflict of Interest}

The authors declare no conflict of interest.

\section{Acknowledgements}

We thank all the participants and officials of Tabriz University Advisory Center who helped us with this research. 
ميداند. اين الكو مطرح ميكند كه ناهنجارىهاى سيستم

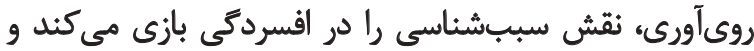

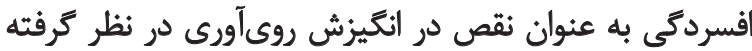

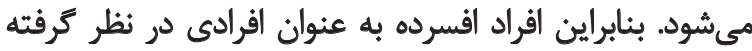

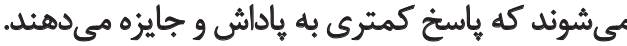
در حمايت از اين نظريه جزندين مطالعه تجربى كزارش كردئد

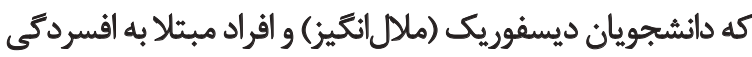

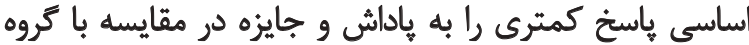

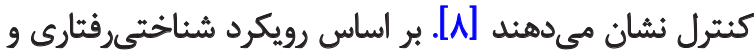

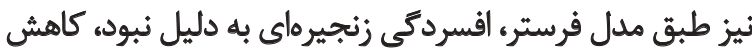

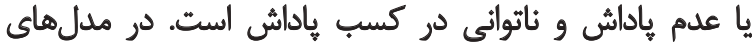

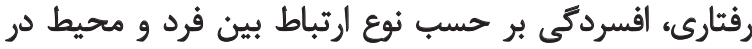

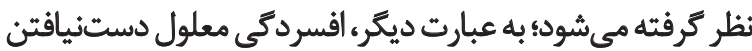

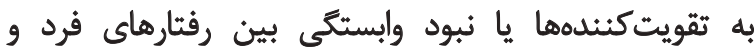
تقويت كنيدههاست [9]]

با توجه به مطالب كفتهشده مي توان كفت كه كسب يُاداش و

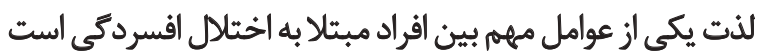

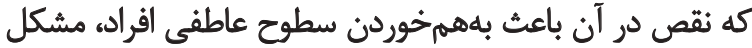

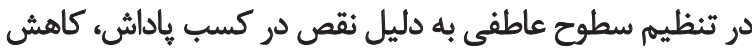

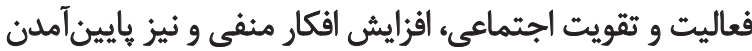

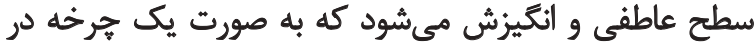

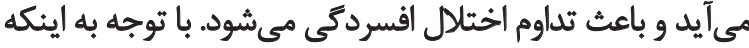

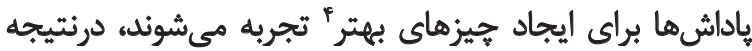

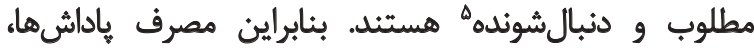
بيامدهاى لذتبخش توليد مى كند كه اين عامل بائ باعث تحكيم

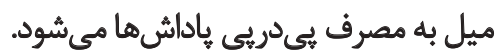
افراد مبتلا به اختلال افسردكى به دليل نقص در رفتارهاي

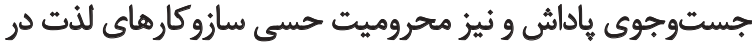

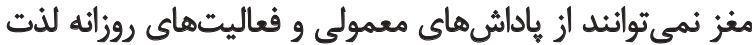

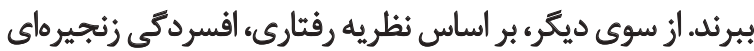

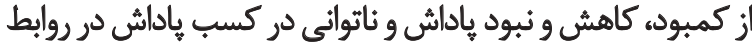

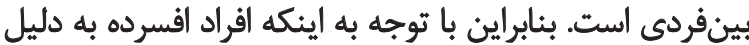

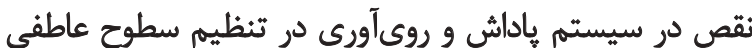

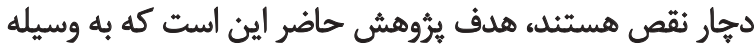

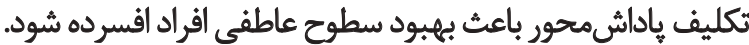

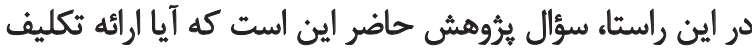

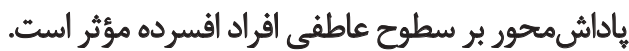

يثروش حاضر مطالعهاى آزمايشى با طرح بيشآزمون و

4. Making things better

5. Pursued

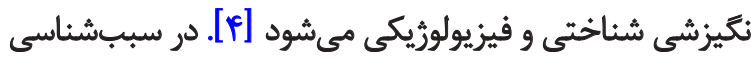

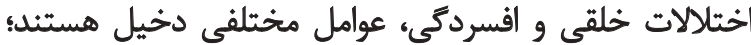

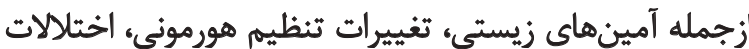

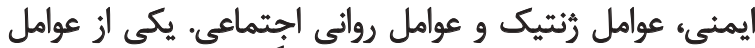

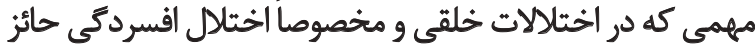

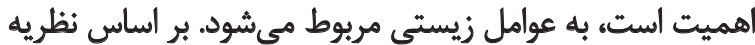

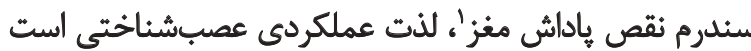

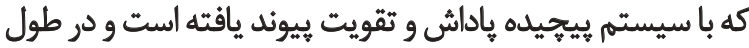

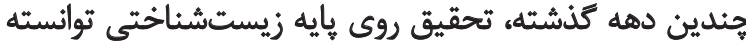

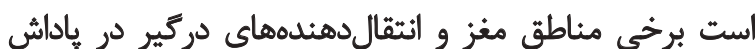

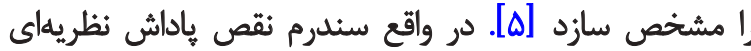

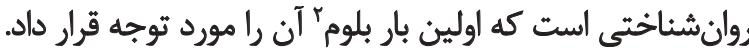

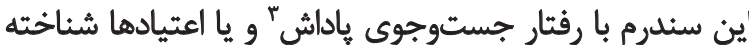

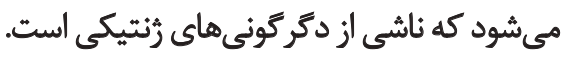

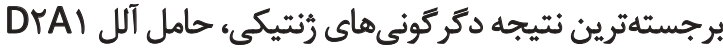

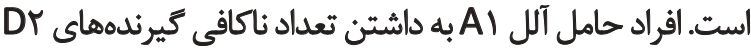

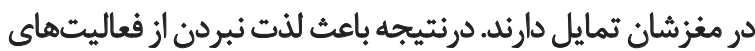

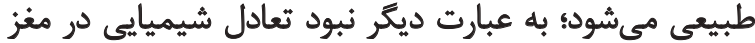

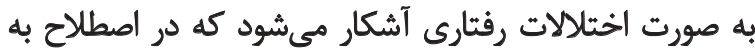

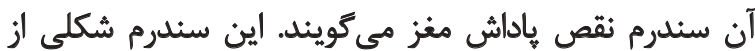

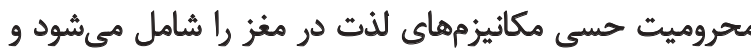

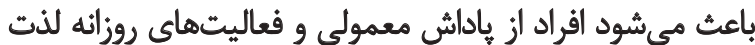

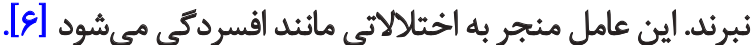

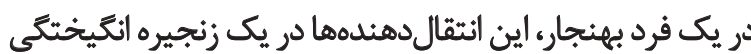

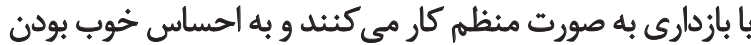

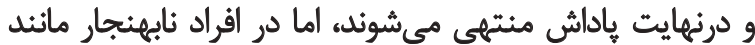

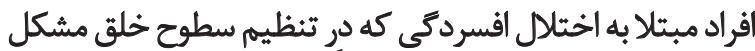

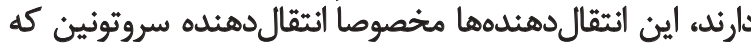

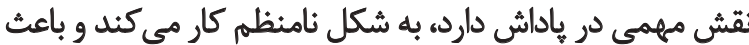

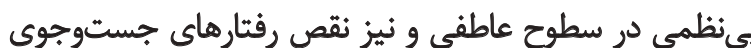

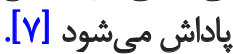

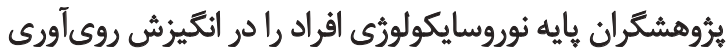

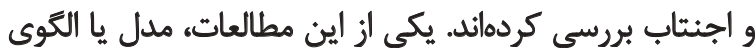

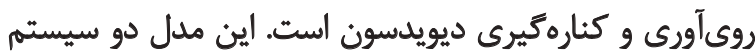

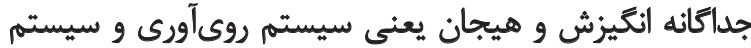

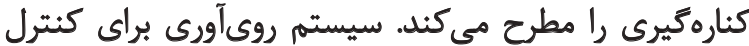

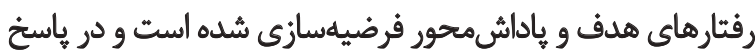

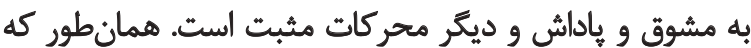

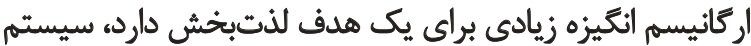
روى آورى اركانيسم رادر برابر توليد عواطف مثبت خاصيت خاصى مسئول

1. Reward deficiency syndrome

2. Kenneth Blum

3. Reward-seeking behavior 


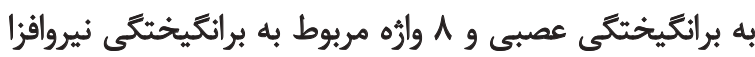

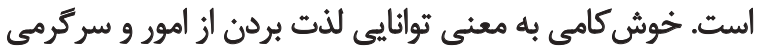

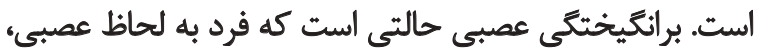

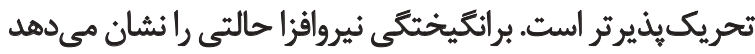

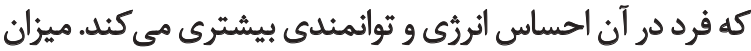

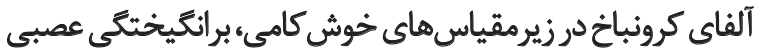

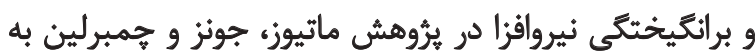

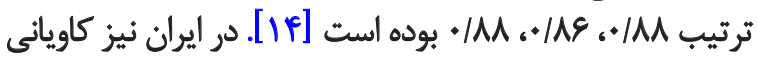

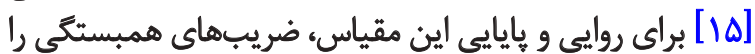

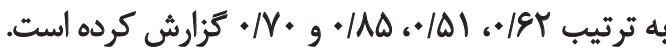

هاخ

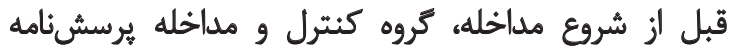

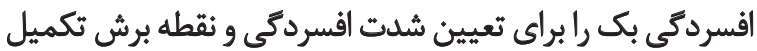

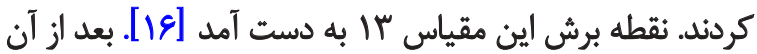

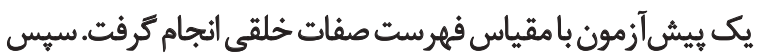

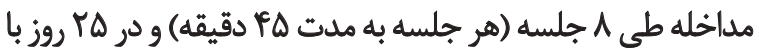

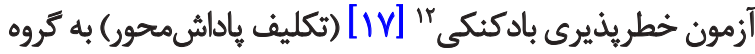

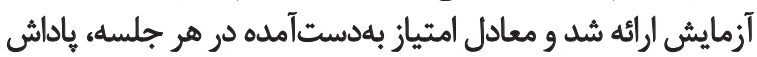

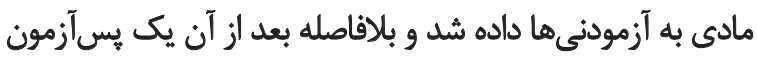

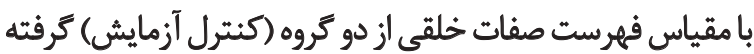

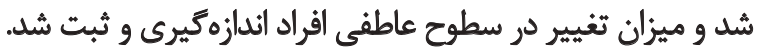

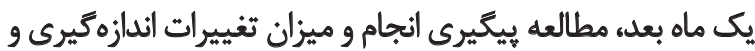

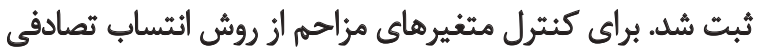

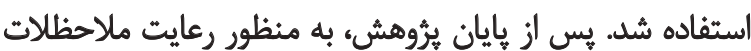
اخلاقى، كروه كنترل نيز تحت مداخله قرار كرفت بئن

آزمون خطريذيرى بادكنكى، نوعى آزمون رايانهاى است كه

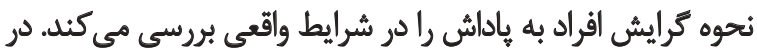

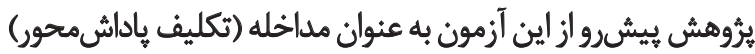

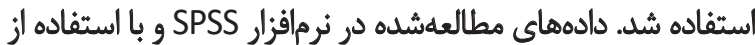

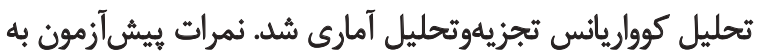

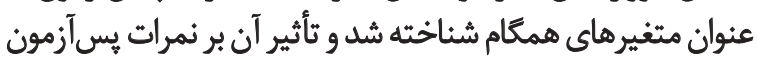
و ييكيرى با استفاده از تحليل كوواريانس بررسي شد.

يس إزبيان اهداف يُروهش براى شركت كنيندكان، رضايت آنها

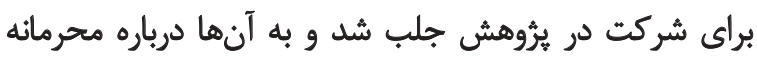

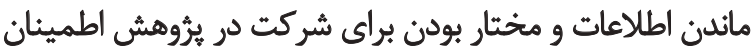

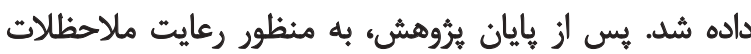
اخلاقي، كروه كنترل نيز تحت مداخله قرار ترفت.

bevill

جدول شماره ا ميانكين و انحراف معيار متغيرهاي مطالعهشده

12. Balloon Analogue Risk Task

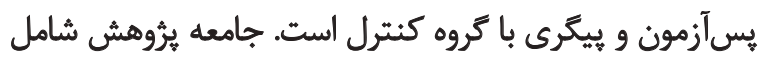

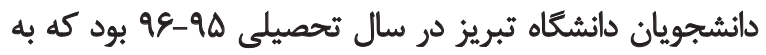

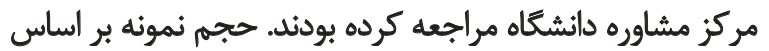

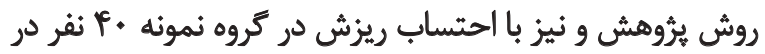

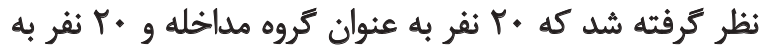

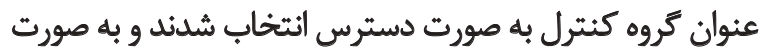

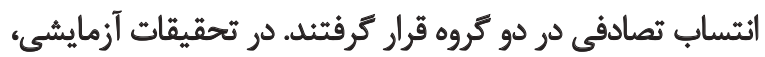

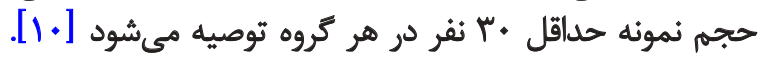

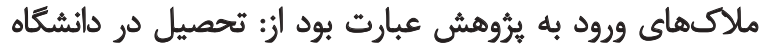

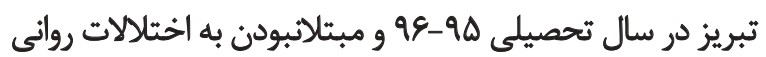

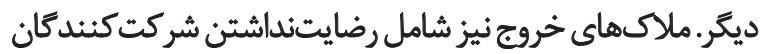

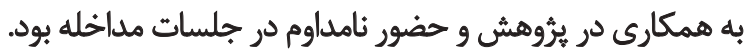

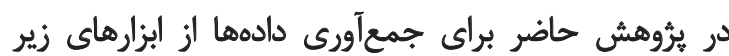

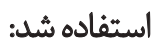

\section{هقياس افسردمَّى بك"}

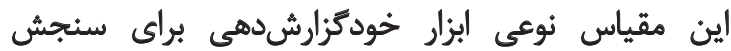

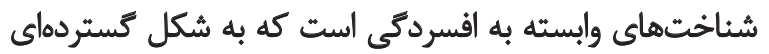

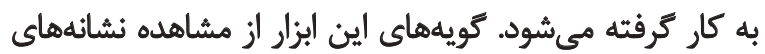

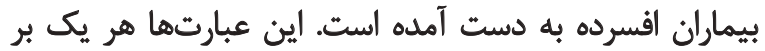

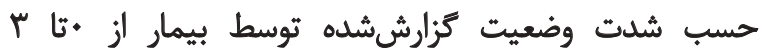

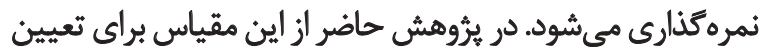

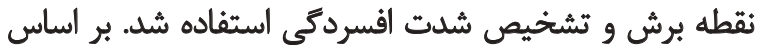

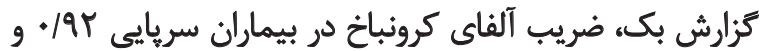

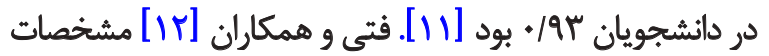

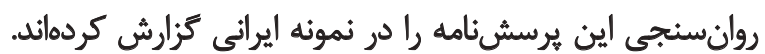

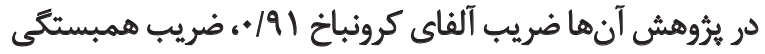

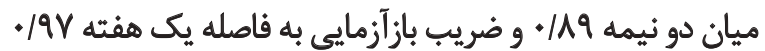

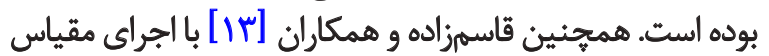

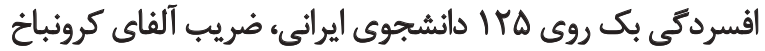

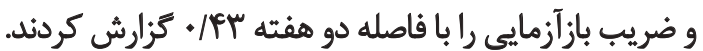

مثياس فُمر ست صفات خلقي"

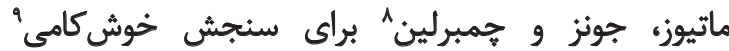

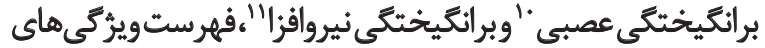

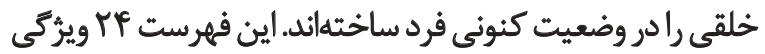

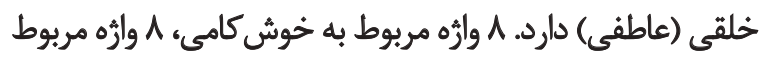

\footnotetext{
6. Beck Depression Inventory (BDI-II)

7. Mood Adjective Check List (MACL)

8. Matthews, Jones, Chamberlain

9. Hedonic tone

10. Tense arousal

11. Energetic arousal
} 


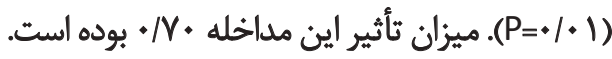

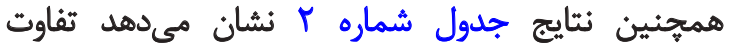

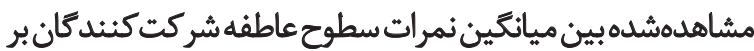

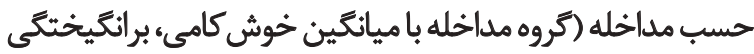

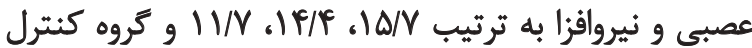

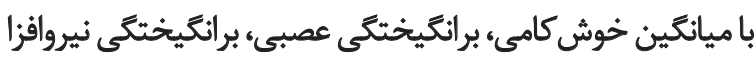

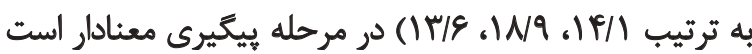

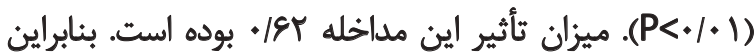

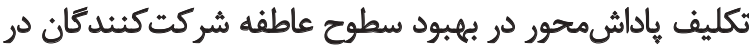

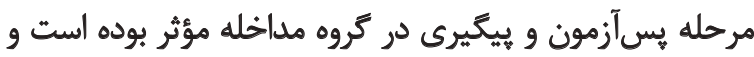

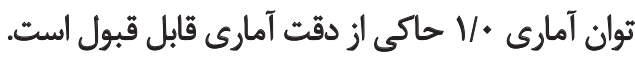

بحث

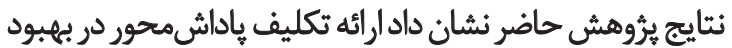

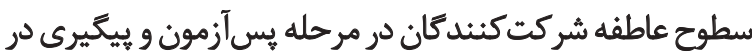

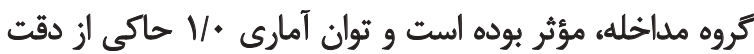

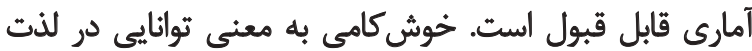

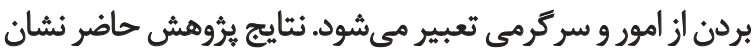

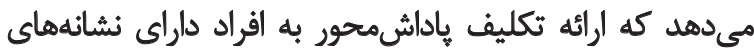

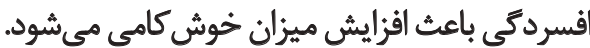
بر اساس مدل فرستر، افسردكى زنجيرهاي به معنى نبود، كاهش

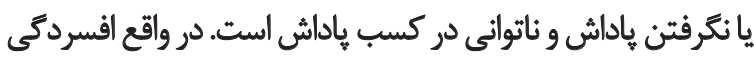

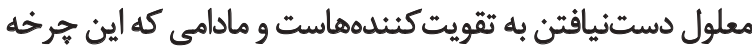

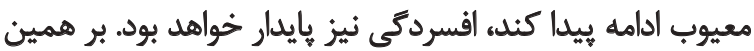

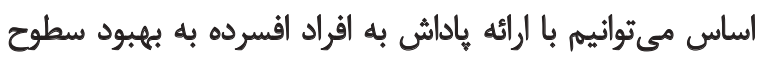

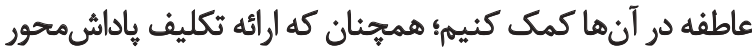

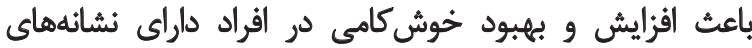

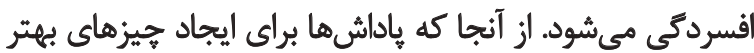

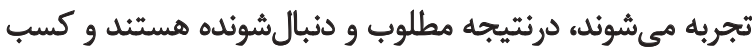

رادر كروهاى مداخله وكنترل در مراحل بيش آزمونء يّآزمون

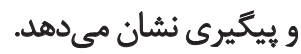

براي همكنى ماتريسهاي كوواريائس از آزمون باكس استفاده

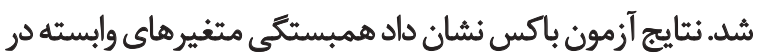

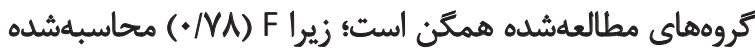

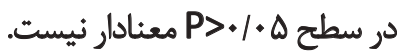

براى بررسى بيشفرض همكنى واريانس خطا از آزمون لون

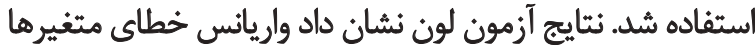

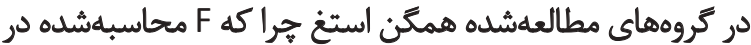

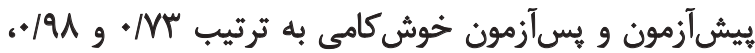

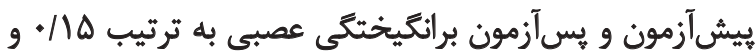

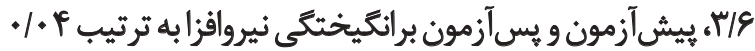
و 1٪٪ است كه در سطح هـ ٪/ معنادار نيست.

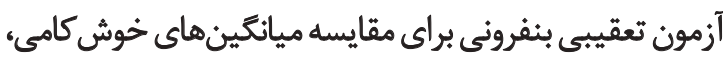

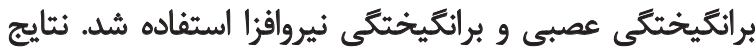

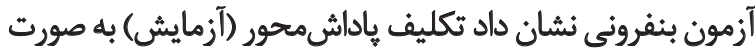

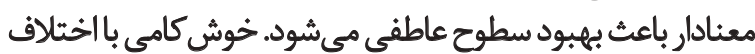

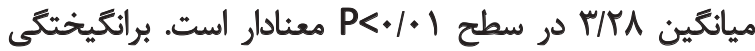

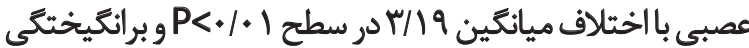

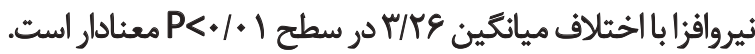
نتايج تحليل كوواريانس و ثأثير مداخله (تكليف باداشمحوري)

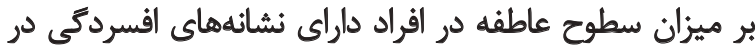

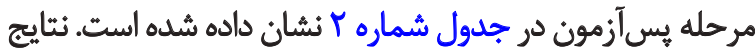

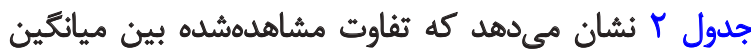

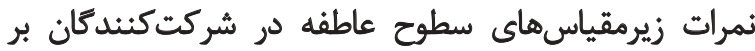

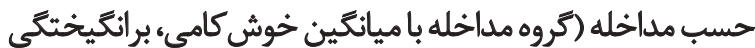

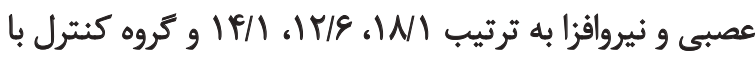

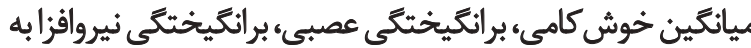

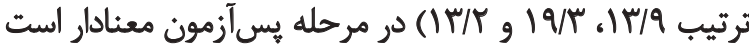

جدول ا. ميانكين و انحراف معيار نمرات سطوح عاطفه در مراحل بيش آزمون، بِ آزمون و يِيكيرى

\begin{tabular}{|c|c|c|c|c|}
\hline بيكيرى (اماه بعد) & يس آزمون & بيش آزمون & \multirow{2}{*}{\multicolumn{2}{|c|}{ كروه }} \\
\hline M(SD) & $M(S D)$ & $M(S D)$ & & \\
\hline $18 / V(T / Q)$ & $W N\left(E / N^{*}\right)$ & $11 / r(r / 1)$ & خوش كامى & \\
\hline $\mid F / e(T / F)$ & $\mid r / g(t / F)$ & $r / r(\Delta / \Delta)$ & برانكيختكى عصبى & آزمايش \\
\hline$W N(r / N)$ & $\mid F / N(\Psi / N)$ & $N \&(Y / I)$ & برانكيختكى نيروافززا & \\
\hline $\mid F /(F / F)$ & $1 w / q(\Delta / 1)$ & $\mid F / T(T / \pi)$ & خوش كامى & \\
\hline$W Q(Y / T)$ & $\mid Q / r(r / A)$ & $|9 /|(r / 9)$ & برانكيختكى عصبى & كتترل \\
\hline $\mid r / g(g / F)$ & $\mid r / T(\xi / \pi)$ & $\mid r / A(\Delta / \Delta)$ & برانكيختكى نيروافزا & \\
\hline
\end{tabular}


جدول Y. نتايج آزمون تحليل كوواريائس تكليف يّاداشمحور بر خوش كامى، برائكيختكى عصبى و نيروافزا

\begin{tabular}{|c|c|c|c|c|c|c|c|}
\hline ثتوان آمارى & ميزان ثأثير & معنادارى & $\mathbf{F}$ & df & شاخص متغير & مراحل & مقياس \\
\hline 1 & .181 & .1 .1 & $M T a / A$ & 1 & ييش آزمون & \multirow{2}{*}{ يس آلزمون } & \multirow{4}{*}{ كل } \\
\hline 1 &.$N$ & .1 .1 & $\Delta \cdot N$ & 1 & ملاخله & & \\
\hline 1 &.$/ 104$ & .1 .1 & $181 \cdot 4$ & 1 & ييش آلزمون & \multirow[b]{2}{*}{ ييكيرى } & \\
\hline 1 & . $18 T$ & .1 .1 & $m / A$ & 1 & ملاخله & & \\
\hline ./9१४ & .10 & .1 .1 & $r \Delta / 1$ & 1 & ييش آزَزمون & \multirow{2}{*}{ يس آزمون } & \multirow{4}{*}{ خوش كامى } \\
\hline.$/ 94$ &.$/ P A$ & .1 .1 & $19 / 0$ & 1 & ملاخله & & \\
\hline - $/ A{ }^{\prime}$ & $\cdot|M|$ & 10.9 & q/or & 1 & بيش آزمون & \multirow{2}{*}{ ييكيرى } & \\
\hline .191 &.$/ M V$ & ..$r$ & $I T / M f$ & 1 & مداخله & & \\
\hline$+/ 941$ & $\cdot 1 \Delta \Delta$ & $+1+1$ & $r E / \Delta$ & 1 & بيش آزنهون & \multirow{2}{*}{ يسآزمون } & \multirow{4}{*}{ برانكيخثني عصبي } \\
\hline.$/ 998$ & - IAT & $.1+1$ & $\pi / 1$ & 1 & مداخله & & \\
\hline $.18 W$ & L & $.1+1$ & S/MT & 1 & ييش آزمهون & \multirow{2}{*}{ بيكيرى } & \\
\hline - $/ A F^{e}$ &.$/ 4 q$ & .1 .1 & $\mid r / 8 A$ & 1 & ملاخله & & \\
\hline .191 &.$/ M V$ & $.1 \cdot \cdot r$ & $\mid r / T$ & 1 & يبش آرنهون & \multirow{2}{*}{ بسآزمون } & \multirow{4}{*}{ برانكيختكى نيروافزا } \\
\hline .144 &.$/ P A$ & .1 .1 & $19 / \pi$ & 1 & ملاخله & & \\
\hline.$/ 4 q$ &.$/ 1 \%$ & .1 .9 & $r / 10$ & 1 & ييش آزنمون & \multirow{2}{*}{ ييكيرى } & \\
\hline " & . & $.10+r$ & $\mid w / \cdot 9$ & 1 & مداخله & & \\
\hline
\end{tabular}

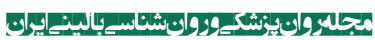

اين بروهش باعث كاهش برانكيختىى عصبى ميى شود.

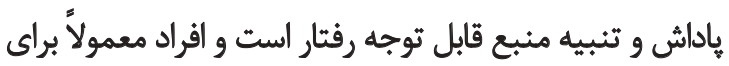

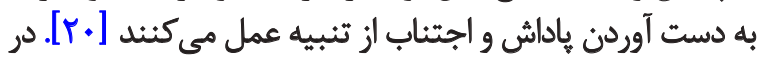

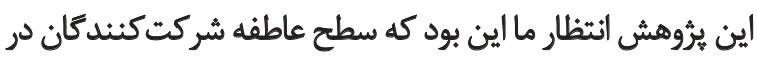

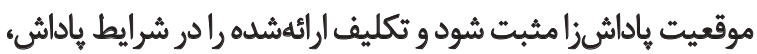

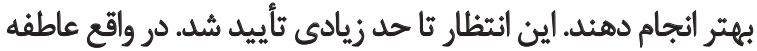

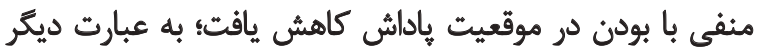

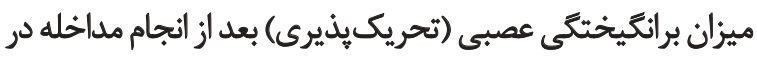

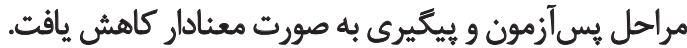
بر اساس نظر وايثر، يُيامدهاى مثبت تمايل دارند كه باسخهاى

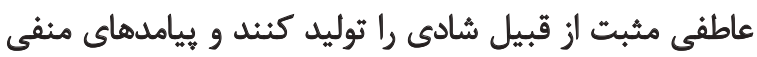

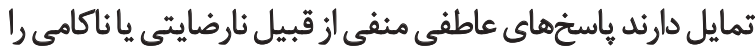

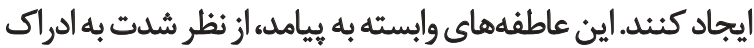

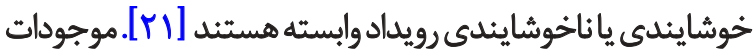

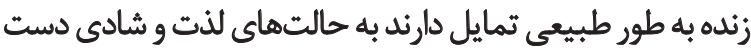

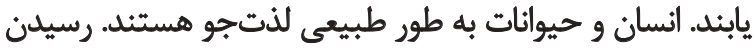

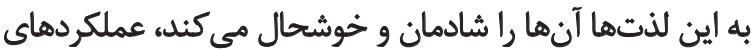

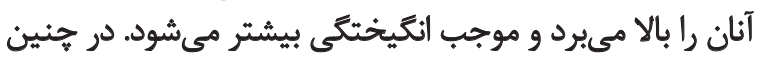

تاداش يامدهاى لذتبخشى توليد مىكند و اين عامل باعث

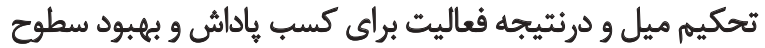

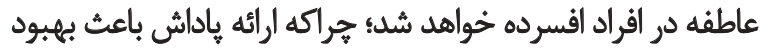

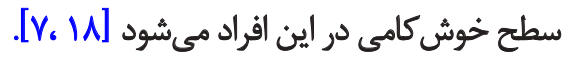
از سوى ديخر با توجه به نظريه سندرم نقص ياداش مغز،

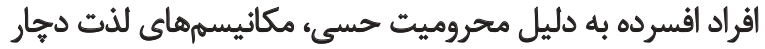

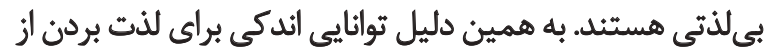

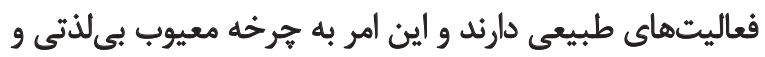

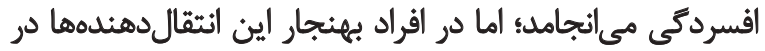

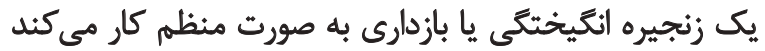

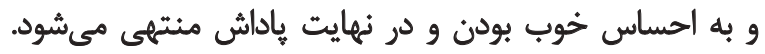

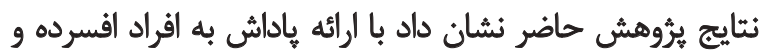

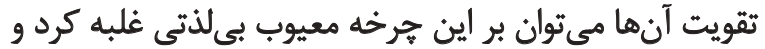

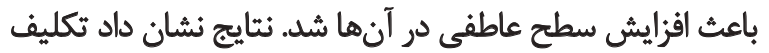

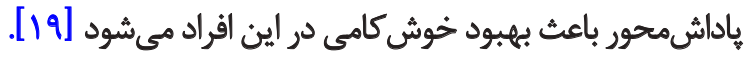
برانكيختكي عصبى حالتي است كه فرد به لحاظ عصبي،

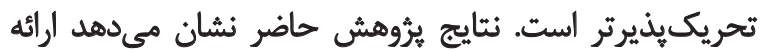

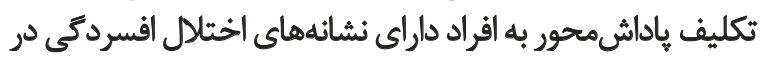


بهبود سطوح عاطفه در افراد افسرده مي مشود و عاطفه منفى با بودن

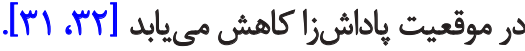

يروهش حاضر روى نمونه غير بالينى و جمعيت دانشجويى اجرا

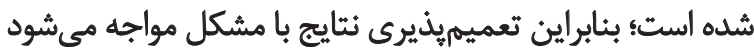

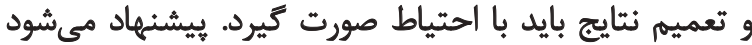

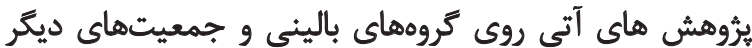

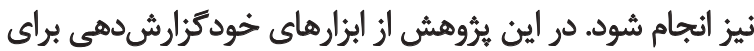

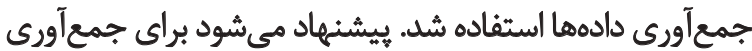

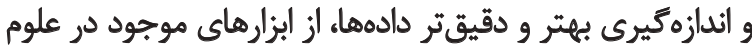
اعصاب شناختى استفاده شود.

$$
\text { سياسكّزازى }
$$

در بايان از همه شركت كنيدكان و مسئولان محترم مركز

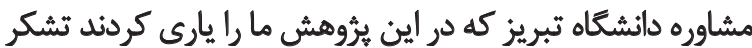

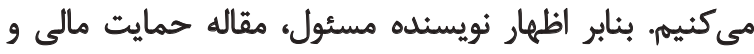

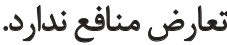

حالى نيرو و اثرثرى بدنى و حتى ذهنى در حلد بهينه قرار مي كيرد

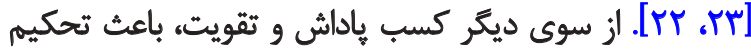

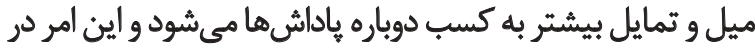

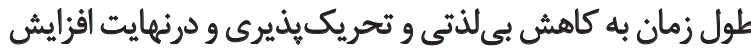

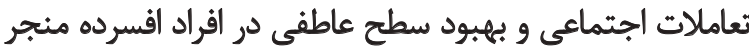

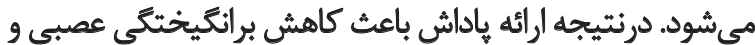

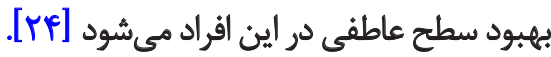

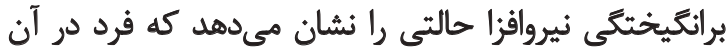

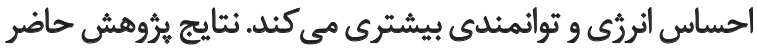

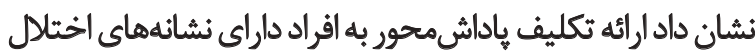

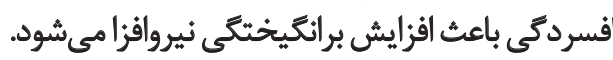

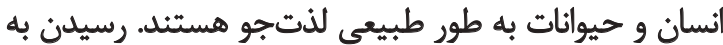

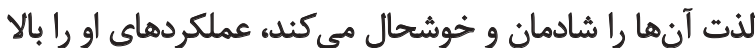

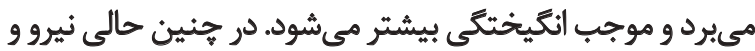

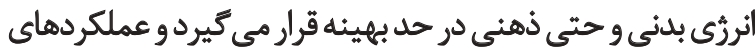

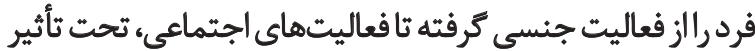

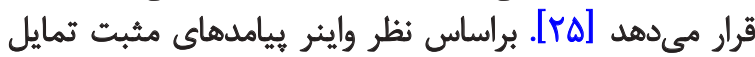

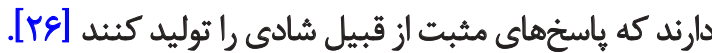

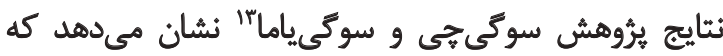

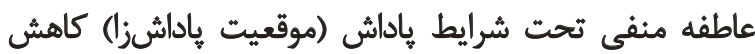

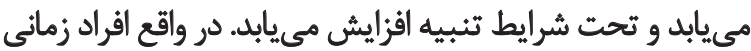

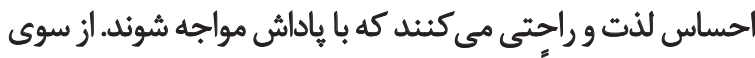

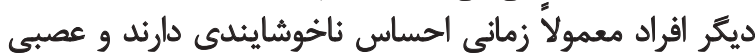

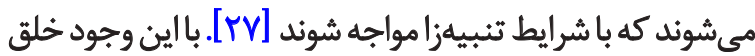

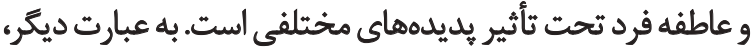

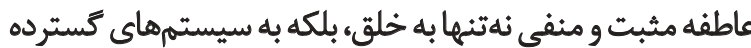

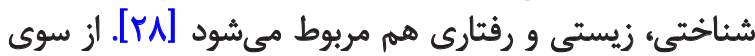

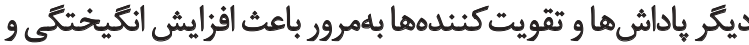

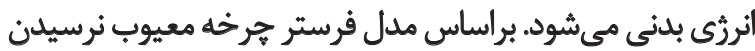

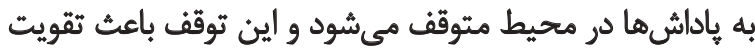

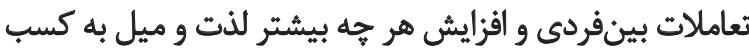

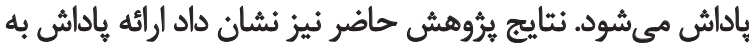

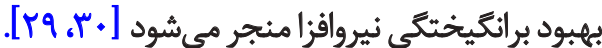
نتيجهنيرى

ارائه تكليف ياداشمحور باعث افزايش و بهبود خوش كامى و

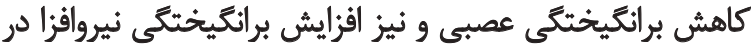

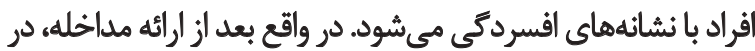

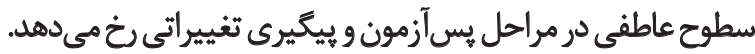

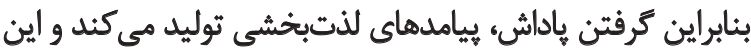

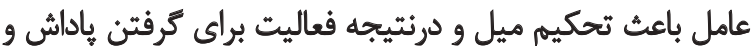

13. Sokichi Sakuragi and Yoshiki Sugiyama 


\section{References}

[1] Kaplan HI, Sadock BJ. Synopsis of psychiatry: Behavioral sciences clinical psychiatry. New York: Williams \& Wilkins Co; 1988.

[2] Chris R Brewin. Cognitive foundation of clinical psychology [MM Alilou, A. Bakhshipour, H. Saboorimoghadam, Persian trans.]. Tabriz: Ravanpoya; 1997.

[3] Zhai L, Zhang Y, Zhang D. Sedentary behaviour and the risk of depression: A meta-analysis. British Journal of Sports Medicine. 2014; 49(11):705-9. doi: 10.1136/bjsports-2014-093613

[4] Blum K, Cull JG, Braverman ER, Comings DE. Reward deficiency syndrome. American Scientist. 1996; 84(2):132-45.

[5] Blum K, Braverman ER, Holder JM, Lubar JF, Monastra VJ, Miller $\mathrm{D}$, et al. The reward deficiency syndrome: A biogenetic model for the diagnosis and treatment of impulsive, addictive and compulsive behaviors. Journal of Psychoactive Drugs. 2000; 32(sup1):1-112. doi: 10.1080/02791072.2000.10736099

[6] Blum K, Meshkin B, Downs BW. DNA based customized nutraceutical "gene therapy" utilizing a genoscore: A hypothesized paradigm shift of a novel approach to the diagnosis, stratification, prognosis and treatment of inflammatory processes in the human. Medical Hypotheses. 2006; 66(5):1008-18. doi: 10.1016/j.mehy.2005.09.029

[7] Davidson RJ. Affective style and affective disorders: Perspectives from affective neuroscience. Cognition \& Emotion. 1998; 12(3):30730. doi: $10.1080 / 026999398379628$

[8] Leahy RL, Holland SJ. Treatment plans and interventions for depression and anxiety disorders [M. Akbari, M. Chiniforoushan, Persian trans.]. Tehran: Arjomand; 2000.

[9] Arias Carrión Ó, Pöppel E. Dopamine, learning, and reward-seeking behavior. Acta Neurobiologiae Experimentalis. 2007; 67(4):481-8. PMIID: 18320725

[10] Delavar A. Research methods in psychology. Tehran: Virayesh Publications; 2006.

[11] Beck AT, Steer RA, Ball R, Ranieri WF. Comparison of Beck Depression Inventories-IA and-II in psychiatric outpatients. Journal of Personality Assessment. 1996; 67(3):588-97. doi: $10.1207 /$ s15327752jpa6703_13

[12] Fata L, Birashk B, Atefvahid MK, Dabson KS. [Meaning assignment structures/schema, emotional states and cognitive processing of emotional information: Comparing two conceptual frameworks (Persian)]. Iranian Journal of Psychiatry and Clinical Psychology. 2005; 11(3):312-26.

[13] Ghassemzadeh H, Mojtabai R, Karamghadiri N, Ebrahimkhani N. Psychometric properties of a Persian-language version of the Beck Depression Inventory - Second edition: BDI-II-PERSIAN. Depression and Anxiety. 2005; 21(4):185-92. doi: 10.1002/da.20070

[14] Matthews G, Jones DM, Chamberlain AG. Refining the measurement of mood: The UWIST Mood Adjective Checklist. British Journal of Psychology. 1990; 81(1):17-42. doi: 10.1111/j.2044-8295.1990. tb02343.x

[15] Kaviani H, Karamghadiri N, Ebrahimkhani N. [Mood swings in different weathers and during seasonal changes in normal population (Persian)]. Iranian Journal of Psychiatry and Clinical Psychology. 2005; 11(2):194-203.

[16] Ahmadian A, Nazari AM, Hatami M, Hassanabadi H, Mirzaei J. [Effectiveness of analogical treatment schema on Dysthymic disorder of veterans with chronic PTSD (Single case experimental schema) (Persian)]. Journal of Military Psychology. 2012;3(11):21-40.
[17] Lejuez CW, Read JP, Kahler CW, Richards JB, Ramsey SE, Stuart GL, et al. Evaluation of a behavioral measure of risk taking: The Balloon Analogue Risk Task (BART). Journal of Experimental Psychology: Applied. 2002; 8(2):75-84. doi: 10.1037/1076-898x.8.2.75

[18] Shankman SA, Klein DN, Tenke CE, Bruder GE. Reward sensitivity in depression: A biobehavioral study. Journal of Abnormal Psychology. 2007; 116(1):95-104. doi: 10.1037/0021-843x.116.1.95

[19] Scott-Parker B, Watson B, King MJ, Hyde MK. A further exploration of sensation seeking propensity, reward sensitivity, depression, anxiety, and the risky behaviour of young novice drivers in a structural equation model. Accident Analysis \& Prevention. 2013 Jan 1;50:465-71.

[20] Sherdell L, Waugh CE, Gotlib IH. Anticipatory pleasure predicts motivation for reward in major depression. Journal of Abnormal Psychology. 2012; 121(1):51-60. doi: 10.1037/a0024945

[21] Bowirrat A, Oscar-Berman M. Relationship between dopaminergic neurotransmission, alcoholism, and reward deficiency syndrome. American Journal of Medical Genetics Part B: Neuropsychiatric Genetics. 2005; 132B(1):29-37. doi: 10.1002/ajmg.b.30080

[22] Chris R Brewin. Cognitive foundation of clinical psychology [MM Alilou, A Bakhshipour, H Saboorimoghadam, Persian trans.]. Tabriz: Ravanpoya; 1997.

[23] Weiner B. Human motivation. New York: Rinehart \& Winston; 2009.

[24] Hashemi T, Andalib Kouraeim M, Pouresmali A, Salehi Heydarabad M. [Effect of inducing mood on cognitive and behavioral performance (Persian)]. Education Strategies in Medical Sciences. 2011; 4(3):101-5

[25] Shankman SA, Nelson BD, Sarapas C, Robison-Andrew EJ, Campbell ML, Altman SE, et al. A psychophysiological investigation of threat and reward sensitivity in individuals with panic disorder and/or major depressive disorder. Journal of Abnormal Psychology. 2013; 122(2):322-38. doi: 10.1037/a0030747

[26] ZajoncA RB. Feeling and thinking: Preferences need no inferences. American Psychologist. 1980; 35(2):151-75. doi: 10.1037/0003-066x.35.2.151

[27] WilliamsA JM, Watts FN, MacLeod C, Mathews A. Cognitive psychology and emotional disorders. Oxford, England: John Wiley \& Sons; 1988.

[28] WatsonA D, Clark LA. Extraversion and its positive emotional core. In: Hogan R, Johnson J, Briggs S, editors. Handbook of Personality Psychology. San Diego: Academic Publication; 1997.

[29] DeciA EL, Ryan RM. Motivation, Personality, and development within embedded social contexts: An overview of self-determination theory. In: Ryan RM. editor. The Oxford Handbook of Human Motivation. Oxford: Oxford University Press; 2012. doi: 10.1093/oxford$\mathrm{hb} / 9780195399820.013 .0006$

[30] SakuragiA S, Sugiyama Y. Effects of reward and punishment on task performance, mood and autonomic nervous function, and the interaction with personality. Journal of Physiological Anthropology. 2009; 28(4):181-90. doi: 10.2114/jpa2.28.181

[31] MartinottiA G, Hatzigiakoumis DS, Vita OD, Clerici M, Petruccelli F, Giannantonio MD, et al. Anhedonia and reward system: Psychobiology, evaluation, and clinical features. International Journal of Clinical Medicine. 2012; 3(7):697-713. doi: 10.4236/ijcm.2012.37125

[32] WillnerA P, Hale AS, Argyropoulos S. Dopaminergic mechanism of antidepressant action in depressed patients. Journal of Affective Disorders. 2005; 86(1):37-45. doi: 10.1016/j.jad.2004.12.010 